Journal of Patient-Centered

Volume 1

Issue 3 -- Cardio-Oncology

Article 6

8-13-2014

\title{
An Overview of Cancer Rehabilitation and Exercise in the Literature: Promoting Increased Referrals to Improve Oncology Outcomes
}

Leslie J. Waltke

Follow this and additional works at: https://aah.org/jpcrr

Part of the Oncology Commons, and the Rehabilitation and Therapy Commons

\section{Recommended Citation}

Waltke LJ. An overview of cancer rehabilitation and exercise in the literature: promoting increased referrals to improve oncology outcomes. J Patient Cent Res Rev. 2014;1:137-141. doi: 10.17294/ 2330-0698.1028

Published quarterly by Midwest-based health system Advocate Aurora Health and indexed in PubMed Central, the Journal of Patient-Centered Research and Reviews (JPCRR) is an open access, peer-reviewed medical journal focused on disseminating scholarly works devoted to improving patient-centered care practices, health outcomes, and the patient experience. 


\title{
An Overview of Cancer Rehabilitation and Exercise in the Literature: Promoting Increased Referrals to Improve Oncology Outcomes
}

\author{
Leslie J. Waltke, PT \\ Cancer Rehabilitation Services, Aurora Health Care, Milwaukee, WI
}

\begin{abstract}
Medical management for malignant neoplastic diseases includes surgery, chemotherapy, radiation, and hormonal and biological therapies. Each of these antineoplastic interventions has unique impacts on physiological, musculoskeletal and functional performance. Cancer rehabilitation is the area of physical medicine responsible for addressing impairments associated with cancer treatment and survivorship, advanced disease and end of life. Although it is expected that more than one-half of persons being treated for cancer will suffer moderate pain and physical and functional decline, and that most will describe fatigue, referrals to rehabilitation are traditionally low. Evidence suggests that referrals to rehabilitation before, during and after treatment for neoplastic disease may improve physiological and functional performance, quality of life and survival outcomes. Oncology practitioners are encouraged to refer patients undergoing cancer treatment to a rehabilitation professional at the peridiagnosis period. (J Patient-Centered Res Rev. 2014;1:137-141.)
\end{abstract}

\section{Keywords}

cancer, fatigue, rehabilitation, radiation, chemotherapy physical therapy, occupational therapy, speech therapy, exercise

\section{Introduction}

Cancer rehabilitation is the area of physical medicine responsible for addressing the musculoskeletal, cardiopulmonary and functional impairments associated with cancer treatment, survivorship, advanced disease and end of life. These services are provided by physical therapists, occupational therapists, speech therapists, audiologists and physiatrists. Common cancer treatment-related adverse long-term and late effects include fatigue, pain, weakness, fibrosis, incontinence, lymphedema, edema, decreased

Correspondence: Leslie J. Waltke, PT, Cancer Rehabilitation

Coordinator, 945 N. 12th Street, Milwaukee, WI, 53233,

Phone: 414-219-7193, Fax: 414-219-7731,

Email: leslie.waltke@aurora.org mobility, impaired cardiopulmonary function, impaired balance, altered gait, safety issues, sexual dysfunction, impaired swallowing and cognitive issues. Although it is expected that more than one-half of persons being treated for cancer will suffer moderate pain and physical and functional decline $^{1}$ and that up to $90 \%$ will describe fatigue, ${ }^{2}$ referrals to rehabilitation are traditionally low. Cheville et al. evidenced this in a 2008 study of 163 women with metastatic breast cancer. Though $92 \%$ of these women were found to have at least one physical impairment requiring rehabilitation intervention, only $30 \%$ received rehabilitative treatment. ${ }^{3}$

Evidence suggests that referrals to rehabilitation before, during and after treatment for neoplastic disease may improve physiological and functional performance, quality of life and survival outcomes. Both the National Accreditation Program for Breast Centers and the American College of Surgeons' Commission on Cancer recognize the importance of cancer rehabilitation and include it as a required standard of an accredited cancer care program. ${ }^{4,5}$ The National Comprehensive Cancer Network (NCCN) also recognizes the benefits of rehabilitation for this population and recommends it begin at cancer diagnosis. ${ }^{2}$ This paper will provide an evidence-based synopsis of the underlying research supporting the referral of patients with cancer to rehabilitation.

\section{Methods}

The studies discussed in this paper were found in the National Library of Medicine. The search strategy was designed to capture studies discussing any type of rehabilitation (physical, occupational or speech therapy) for any type of cancer. A broad search of the national and international body of evidence in cancer rehabilitation and cancer and exercise was performed within the Ovid/Medline database from 2004 to 2014 for pertinent articles in English. The key search words were cancer, fatigue, radiation, chemotherapy, rehabilitation, physical therapy, occupational therapy, speech therapy and exercise. This search resulted in more than 200 pertinent articles. Common themes of study results emerged, falling into several categories including: 
1) preintervention rehabilitation decreased postsurgical and postradiation morbidity, 2) exercise during chemotherapy and radiation treatment decreased acute and delayed fatigue and functional loss, 3) rehabilitation improved postoperative outcomes, and 4) exercise after cancer treatment increased quality of survivorship and increased length of life. This narrative review will highlight studies from each of those categories. Of note, none of the studies found appropriate rehabilitation or exercise interventions to be detrimental to the patient with cancer or a history of cancer.

\section{Exercise as Treatment}

Evidence supporting the use of exercise in prolonging life and in the treatment and prevention of disease has a robust and long history. Even the ancient Greek physician Hippocrates noted that "bodies grow relaxed and squat... through their sedentary lives," resulting in one of his most famous quotes: "walking is man's best medicine."

In 2012, the National Cancer Institute released results from a large observational exercise study of 650,000 subjects from the general population, controlled for both tobacco and alcohol use. For individuals over the age of 40 years, researchers found increases in life expectancy from walking ranging from 1.8 years for walking 75 minutes per week to 4.5 years of added life for walking 450 minutes per week. Notably, increased lifespan was seen for all persons exercising, regardless of body mass index. ${ }^{6}$

Rehabilitation and exercise have been encouraged in patients with vascular, cardiac, pulmonary, neurological and orthopedic impairments for decades with good result. Unfortunately, though evidence suggests that exercise and rehabilitation during and after oncology medical management may improve quality of life, prolong life, reduce the risk of metastasis and decrease deaths from cancer, "walking as medicine" is not routinely being applied to the patient with cancer. Multiple studies indicate that cancer survivors are less active than the general population, ${ }^{7}$ and most do not meet the activity guidelines of the American Cancer Society. ${ }^{8}$ Although much of the cancer rehabilitation and exercise research has been done on patients with breast cancer, studies promoting the use of rehabilitation and exercise for the patient with other types of primary cancer are prevalent. As a result, an opportunity to optimize health and outcomes in the cancer population with well-documented cardiopulmonary, musculoskeletal and functional decline is being missed.

\section{Pre-Cancer Treatment Rehabilitation}

Prehabilitation, by definition, is the use of rehabilitation prior to a medical intervention. ${ }^{9}$ The use of preoperative rehabilitative training and teaching in orthopedic medicine is common. Evidence shows that patients receiving physical therapy prior to orthopedic surgery have better outcomes and shortened postoperative hospital stays. This evidence also is available in various types of primary cancers. For example, a randomized trial by Mayo et al. showed patients with colorectal cancers that responded to preoperative stationary cycling and weight training were more likely to recover to their baseline capacity and were at lesser risk of postsurgical complications requiring intensive care or further surgical intervention. ${ }^{10}$ For patients with operable lung cancers, a 2011 study demonstrated that undergoing preoperative physical therapy decreased the length of postoperative hospital stays and increased both oxygen saturation and exercise capacity. ${ }^{11}$

Studies also advocate preoperative rehabilitation intervention for men with prostate cancer. Those undergoing radical prostatectomy and receiving exercise and pelvic floor training preoperatively have fewer issues with postoperative incontinence. ${ }^{12}$ Benefits of pre-cancer treatment rehabilitation intervention are found in radiation therapy as well. Patients receiving radiation for head and neck cancers are known to be at high risk for developing dysphagia. With preradiation speech therapy, swallow function is improved, patients suffer less dietary change and stenosis, and fewer require gastrostomy feeding tube placements resulting from the radiation. ${ }^{13}$

\section{Rehabilitation and Exercise During Systemic Cancer Treatment}

Systemic cytotoxic and cytostatic treatment for cancer is known to cause a plethora of negative side effects, including fatigue, cardiomyopathy, excess weight loss, weight gain, anemia, arthralgias and muscle mass loss. These side effects often result in negative physiological change and functional loss. The NCCN reports fatigue is the most frequent side effect of cancer treatment, describing its frequency as "nearly universal," and defines cancer-related fatigue as "a distressing, persistent, subjective sense of physical, emotional and/or cognitive tiredness or exhaustion related to cancer or cancer treatment that is not proportional to recent activity and interferes with usual functioning."2 For patients undergoing chemotherapy and radiation, rehabilitation treatment programs that include the upper and lower extremities, core strengthening and prescriptive aerobic exercise have been shown to lessen fatigue, sarcopenia 
(loss of skeletal muscle mass) and anemia. ${ }^{14}$ Specifically for radiation, Druin et al. showed in their 2006 study that moderate aerobic exercise during chest wall radiation in women with breast cancer maintained erythrocyte levels in the blood stream, which led to less fatigue and improved peak aerobic capacity. ${ }^{15}$

Doxorubicin is an anthracycline antibiotic used in the treatment of many types of cancers that has well-documented cardiomyopathy effects. Evidence exists that exercise may preserve cardiac function and reduce myocardial oxidative stress due to doxorubicin. Though mechanisms of action are not fully clear, research studies show aerobic exercise reduces activation of proapoptotic signaling and increases GATA-4 expression, leading to preservation of cardiomyocyte proliferation, improved calcium handling and activation of the AMP-activated protein kinase pathway. ${ }^{16}$

Exercising patients with low blood counts may raise concern. However, with modifications to protect the patient from risks of infection from neutropenia or bleeding from thrombocytopenia, rehabilitation and exercise in hospital inpatients with severe cytopenia has been shown to be safe. ${ }^{17}$ Groeneveldt et al. placed patients with multiple myeloma on rehabilitation programs consisting of resistance, stretching and aerobic exercise. ${ }^{18}$ About $80 \%$ of patients asked to join the study consented, and $73 \%$ of those who participated adhered to independent exercise. Improvements in quality-of-life measures, fatigue scores, and upper and lower extremity strength were seen. Though some patients were removed from the study due to disease progression and fracture risk, no adverse events were noted in the rehabilitation group, leading the authors to conclude that exercise programs in patients with multiple myeloma are "feasible and safe." 18 Also of great importance, Courneya and colleagues found that patients exercising during chemotherapy for breast cancer maintained their 21-day cycle of delivery more frequently than nonexercisers, potentially leading to improved survival outcomes. ${ }^{19}$

\section{Rehabilitation to Improve Postoperative Function}

Surgical hepatectomy due to hepatocellular carcinoma can have a significant impact on postoperative function. In a 2013 study of posthepatectomy patients randomized to diet therapy and exercise, Kaibori et al. reported that exercising at the anaerobic threshold over 6 months led to improvements in whole body mass, fasting serum insulin, anaerobic threshold and peak oxygen consumption. ${ }^{20}$ In a study involving 58 patients with colorectal cancers, Djurasić et al. found shorter hospital stays and improvements in functional postoperative recovery, quality of life, and physical and mental health with rehabilitation. ${ }^{21}$ For women requiring surgery for breast cancer, postoperative rehabilitation has been shown to eliminate pain, restore shoulder mobility and strength and improve quality of life compared to controls. ${ }^{22}$ Lymphedema is a chronic postoperative condition feared by many women who require lymph node biopsy. By improving the remaining lymphatic function through restoring full preoperative function at the surgical upper quarter (chest wall, axilla and shoulder), physical therapy was found to decrease the risk of lymphedema in breast cancer patients after undergoing lymph node biopsy. ${ }^{23}$ Weightlifting in breast cancer survivors with and without lymphedema also has been shown to be both safe and beneficial. ${ }^{24}$

\section{Rehabilitation and Exercise in Cancer Survivorship}

It is estimated that there are more than 13 million cancer survivors living in the United States today. ${ }^{25}$ Multiple studies suggest obesity is negatively linked to breast cancer survivorship. Obesity in breast cancer is linked to metastatic recurrence, decreased disease-free interval and increased mortality. ${ }^{26}$ In a 2009 paper, Nichols et al. indicated that every $5-\mathrm{kg}$ weight gain in breast cancer survivors is associated with an increased breast cancer mortality of $13 \% .{ }^{27}$ Conversely, cancer outcomes, quality of life and prognosis are negatively impacted by excessive weight loss and cachexia. Therapeutic exercise in a rehabilitative setting has been shown to slow the rate of functional loss in patients with cachexia by increasing physical activity and improving the balance between muscle protein breakdown and syntheses. ${ }^{28}$ Arthralgias are common with aromatase inhibitors used in postmenopausal breast cancer antiestrogen management. A study published in 2012 demonstrated that physical therapy and exercise may decrease the musculoskeletal pain and loss associated with estrogen-deprivation therapies. ${ }^{29}$

Rehabilitation and exercise in cancer survivorship also may contribute to the actual prevention of cancer deaths and morbidity. Holmes et al. found that for women with stage I to stage III breast cancer, moderate exercise decreases the risk of death from breast cancer by $19 \%$ for walking 1-3 hours per week and by 54\% for walking 3-5 hours per week. ${ }^{30}$ A Duke Cancer Institute study showed that in patients with advanced recurrent glioma, 30 minutes of brisk exercise 5 days a week not only decreased treatmentrelated symptoms but increased survival from 13.03 months 
to 21.84 months. Also of note, the exercise capacity of these patients was additional helpful data for physicians in determining patients' life expectancies. ${ }^{31}$

\section{Discussion}

Surgery, radiotherapy, and cytotoxic and cytostatic treatments for cancer are associated with pain, fatigue, decreased quality of life and decreased life expectancy. The current body of evidence supports using physiatry, physical therapy, occupational therapy and speech therapy in assisting patients undergoing treatment for neoplastic disease in preventing, reducing and eliminating physiological, musculoskeletal and functional decline. From the local functional limitations due to surgery and radiation to the systemic dysfunctions from chemotherapy and hormonal and biological therapies, a robust amount of literature exists confirming that rehabilitation and exercise is safe and beneficial when done under the care of a trained rehabilitation therapist. Still, cancer rehabilitation is underutilized. In its 2014 clinical guidelines for the treatment of cancer-related fatigue, ${ }^{2}$ the NCCN recommends referral to physical therapy in cancer patients with comorbidities, functional or anatomical deficits, substantial deconditioning, and in those requiring surgeries. Since most patients receiving treatment for malignant neoplastic disease will typically suffer one or more physical or functional deficits, the NCCN also recommends that rehabilitation begin at the time of diagnosis. ${ }^{2}$

In addition, with the Affordable Care Act incentivizing shorter length of hospital stays and fewer hospital readmissions and emergency department visits, expanding the practice patterns of oncology clinicians to incorporate cancer rehabilitation would be expected to result in decreased medical costs while improving outcomes. Referral at the time of diagnosis versus later in the treatment path provides the opportunity to minimize loss, maintain function, promote a faster recovery posttreatment and provide an immediate trajectory to improve survival.

\section{Conclusion}

A well-researched, insurance-covered modality to decrease morbidity and mortality associated with cancer treatment and cancer survivorship is available in the avenue of cancer rehabilitation. Oncology practitioners are encouraged to refer patients undergoing cancer treatment to a rehabilitation professional at the peridiagnosis period. Patients should then receive a rehabilitation assessment, treatment as indicated, and be followed or overseen by the rehabilitation practitioner throughout local and systemic treatment and into survivorship. Regardless of the oncological nature of the surgery, systemic therapy or radiation, the resulting common and detrimental dysfunctions respond well to rehabilitation in many types of primary cancers. Because increased patient satisfaction, fewer adverse effects and improved oncology outcomes are associated with cancer rehabilitation, referral to rehabilitation at the time of cancer diagnosis is warranted and encouraged.

\section{Conflicts of Interest}

None.

\section{References}

1. Marcus DA. Epidemiology of cancer pain. Curr Pain Headache Rep. 2011;15:231-4.

2. National Comprehensive Cancer Network. NCCN Clinical Practice Guidelines in Oncology: Cancer-Related Fatigue, Version 1.2014. Available at: www.nccn.org/professionals/physician_gls/pdf/ fatigue.pdf. Accessed May 30, 2014.

3. Cheville AL, Troxel AB, Basford JR, Kornblith AB. Prevalence and treatment patterns of physical impairments in patients with metastatic breast cancer. J Clin Oncol. 2008;26:2621-9.

4. National Accreditation Program for Breast Centers. NAPBC components. Available at: www.napbc-breast.org/standards/ standards.html. Accessed May 30, 2014.

5. American College of Surgeons Commission on Cancer. Cancer program and data standards. Available at: www.facs.org/cancer/ coc/standards.html. Accessed May 30, 2014.

6. Moore SC, Patel AV, Matthews CE, et al. Leisure time physical activity of moderate to vigorous intensity and mortality: a large pooled cohort analysis. PLoS Med. 2012;9:e1001335.

7. Vance V, Mourtzakis M, McCargar L, Hanning R. Weight gain in breast cancer survivors: prevalence, pattern and health consequences. Obes Rev. 12: 282-94.

8. Bellizzi KM, Rowland JH, Jeffery DD, McNeel T. Health behaviors of cancer survivors: examining opportunities for cancer control intervention. J Clin Oncol. 2005;23:8884-93.

9. Silver JK, Baima J. Cancer prehabilitation: an opportunity to decrease treatment-related morbidity, increase cancer treatment options, and improve physical and psychological health outcomes. Am J Phys Med Rehabil. 2013;92:715-27.

10. Mayo NE, Feldman L, Scott S, et al. Impact of preoperative change in physical function on postoperative recovery: argument supporting prehabilitation for colorectal surgery. Surgery. 2011;150:505-14.

11. Pehlivan E, Turna A, Gurses A, Gurses HN. The effects of preoperative short-term intense physical therapy in lung cancer patients: a randomized controlled trial. Ann Thorac Cardiovasc Surg. 2011;17:461-8.

12. Centemero A, Rigatti L, Giraudo D, et al. Preoperative pelvic floor muscle exercise for early continence after radical prostatectomy: a randomised controlled study. Eur Urol. 2010;57:1039-43.

13. Duarte VM, Chhetri DK, Liu YF, Erman AA, Wang MB. Swallow preservation exercises during chemoradiation therapy maintains swallow function. Otolaryngol Head Neck Surg. 2013;149:878-84.

14. Silver JK, Baima J, Mayer RS. Impairment-driven cancer rehabilitation: an essential component of quality care and survivorship. CA Cancer J Clin. 2013;63:295-317.

15. Drouin J, Young TJ, Beeler J, et al. Randomized control clinical trial on the effects of aerobic exercise training on erythrocyte levels during radiation treatment for breast cancer. Cancer. 2006; 107:2490-5.

16. Chicco AJ, Schneider CM, Hayward R. Exercise training attenuates acute doxorubicin-induced cardiac dysfunction. I Cardiovasc Pharmacol. 2006;47:182-9. 
17. Elter T, Stipanov M, Heuser E, et al. Is physical exercise possible in patients with critical cytopenia undergoing intensive chemotherapy for acute leukaemia or aggressive lymphoma? Int $\mathrm{J}$ Hematol. 2009;90:199-204.

18. Groeneveldt L, Mein G, Garrod R, et al. A mixed exercise training programme is feasible and safe and may improve quality of life and muscle strength in multiple myeloma survivors. BMC Cancer. 2013;13:31.

19. Courneya KS, Segal RJ, Mackey JR, et al. Effects of aerobic and resistance exercise in breast cancer patients receiving adjuvant chemotherapy: a multicenter randomized controlled trial. J Clin Oncol. 2007;25:4396-404.

20. Kaibori M, Ishizaki M, Matsui K, et al. Perioperative exercise for chronic liver injury patients with hepatocellular carcinoma undergoing hepatectomy. Am J Surg. 2013;206:202-9.

21. Djurasić L, Pavlović A, Zarić N, Palibrk I, Basarić D, Djordjević VR. The effects of early rehabilitation in patients with surgically treated colorectal cancer. Acta Chir Iugosl. 2012;59:89-91.

22. Galantino ML, Stout NL. Exercise interventions for upper limb dysfunction due to breast cancer treatment. Phys Ther. 2013;93:1291-7.

23. Torres Lacomba M, Yuste Sánchez MJ, Zapico Goñi A, et al. Effectiveness of early physiotherapy to prevent lymphoedema after surgery for breast cancer: randomized, single blinded, clinical trial. BMJ. 2010;340,b5396.

24. Schmitz KH, Ahmed RL, Troxel A, et al. Weight lifting in women with breast cancer-related lymphedema. New Engl J Med. 2009;361:664-73.
25. American Cancer Society. Survivorship and quality of life research. Available at: http://www.cancer.org/research/ survivaltreatmentresearch/index. Accessed May 30, 2014.

26. Ellsworth RE, Valente AL, Shriver CD, Bittman B, Ellsworth DL. Impact of lifestyle factors on prognosis among breast cancer survivors in the USA. Expert Rev Pharmacoecon Outcomes Res. 2012;12:451-64.

27. Nichols HB, Trentham-Dietz A, Egan KM, et al. Body mass index before and after breast cancer diagnosis: associations with allcause, breast cancer, and cardiovascular disease mortality. Cancer Epidemiol Biomarkers Prev. 2009;18:1403-9.

28. Maddocks M, Murton AJ, Wilcock A. Improving muscle mass and function in cachexia: non-drug approaches. Curr Opin Support Palliat Care. 2011;5:361-4.

29. Jones C, Gilmore J, Saleh, M, Feinberg B, Kissner M, Simmons SJ. Therapeutic optimization of aromatase inhibitor-associated arthralgia: etiology, onset, resolution, and symptom management in early breast cancer. Commun Oncol. 2012;9:94-101

30. Holmes MD, Chen WY, Feskanich D, Kroenke CH, Colditz GA. Physical activity and survival after breast cancer diagnosis. JAMA. 2005;293:2479-86.

31. Ruden E, Reardon DA, Coan AD, et al. Exercise behavior, functional capacity, and survival in adults with malignant recurrent glioma. J Clin Oncol. 2011;29:2918-23.

(C) 2014 Aurora Health Care, Inc. 